\title{
Colony-stimulating factors for chemotherapy-related febrile neutropenia are associated with improved prognosis in adult acute lymphoblastic leukemia
}

\author{
SHI-GUANG YE* , YI DING* , LIANG LI, MENG YANG, WEN-JUN ZHANG and AI-BIN LIANG \\ Department of Hematology, Tongji Hospital, Tongji University School of Medicine, Shanghai 200092, P.R. China
}

Received December 15, 2014; Accepted February 20, 2015

DOI: $10.3892 / \operatorname{mco} .2015 .527$

\begin{abstract}
Colony-stimulating factors (CSF) have been widely used to prevent febrile neutropenia associated with chemotherapy. Due to the high intensity of chemotherapy in acute lymphoblastic leukemia (ALL), CSF as a crucial component of supportive care has played a significant role in the therapy. However, the effectiveness of CSF in treatment has not been identified by large clinical trials until now. The aim of the present study was to evaluate the effect of CSF on the long-term outcome of adult ALL patients. A comprehensive search strategy has been conducted, which covered the Cochrane Central Register of Controlled Trials, PubMed and Web of Science. The result includes seven randomized controlled trials containing a total of 753 patients. The administration of CSF significantly reduced the mortality at the end of the follow-up (RR, 0.85; 95\% CI, 0.75-0.95), the mortality at day 30 (RR, 0.41; 95\% CI, 0.23-0.74) and the number of patients with infection or severe infections (RR, 0.8; 95\% CI, 0.7-0.9 and RR, 0.48; 95\% CI, 0.3-0.75). The addition of CSF also marginally increased the number of patients achieving CR (RR, 1.14; 95\% CI, 1.05-1.23). The use of CSF also shortened the duration of neutropenia (median days, 8-17 to 12.5-24). In conclusion, CSFs can be administered to ALL patients during myelosuppressive chemotherapy, particularly in the induction phase.
\end{abstract}

\section{Introduction}

Acute lymphoblastic leukemia (ALL) is a heterogenous hematological disease characterized by the proliferation of immature lymphoid cells in the bone marrow, peripheral blood, and other

Correspondence to: Dr Wen-Jun Zhang or Professor Ai-Bin Liang, Department of Hematology, Tongji Hospital, Tongji University School of Medicine, 389 Xincun Road, Shanghai 200092, P.R. China E-mail: zhangwenjun@tongji.edu.cn

E-mail: lab7182@tongji.edu.cn

${ }^{*}$ Contributed equally

Key words: colony-stimulating factors, acute lymphoblastic leukemia, meta-analysis, survival organs (1). Statistically, the incidence rate of ALL in the USA is 1.6/100,000 individuals per year (2). There are 6,000 estimated new cases (male:female prevalence of 1.3:1) of ALL diagnosed yearly in the USA. Patients are mainly children; $~ 60 \%$ of cases occur in people aged $<20$ years (3). ALL represents $75-80 \%$ of paediatrics acute leukemia, and by contrast, it only represents $20 \%$ of all adult leukemia (4). Over the past several decades, the cure rate and survival outcome of ALL have been significantly improved, particularly among children with ALL. In the current treatment therapy, the complete remission (CR) among children with ALL is 80\% (5-7); but in adults with ALL, the long-term prognosis with CR is $\sim 30-40 \%$ (8-12). The difference between children and adults in long-term outcomes can be explained partly by the difference in cytogenetic subtypes of ALL among age groups (13-15).

Colony-stimulating factors (CSF) are a family of cytokines, which can regulate the proliferation and differentiation of hematopoietic cells. Currently, there are $>20$ molecules regarded as CSFs (16). Among them, granulocyte CSF (G-CSF) and granulocyte macrophage CSF (GM-CSF) have been used clinically. G-CSF regulates the production of neutrophil lineage. Administration of G-CSF results in a dose-dependent increase in circulating neutrophils $(16,17)$. GM-CSF is a growth factor that stimulates the granulocytes, macrophages and eosinophil colonies. Administration of GM-CSF results in a dose-dependent increase in blood neutrophils, eosinophils, macrophages and occasionally lymphocytes $(16,17)$.

Administration of CSF can be used in the following ways: i) Priming of chemotherapy or bone marrow transplantation. The purpose of the former is to recruit leukemic cells into the cell cycle and enhance the effect of chemotherapy, the aim of the latter is to mobilize the hematopoietic cells to the periphery and collect stem cells; ii) during or following chemotherapy so as to accelerate the hematopoietic cells proliferation and decrease the incidence of febrile neutropenia infection; and iii) during febrile neutropenia in order to reinforce the recovery of infection. The first way was excluded in the present study, as the aim was to evaluate the prophylactic effect of CSF (18).

Thus far there is no conclusive data to ensure the effectiveness of CSF to prevent myelosuppressive therapy-related infectious complications. In addition, no evidence shows that the addition of CSF decreases the mortality in adult ALL patients and improves the outcomes. Therefore, the present systematic review was conducted to evaluate the safety and 
effectiveness of the addition of G-CSF or GM-CSF to chemotherapy in adult ALL patients.

\section{Materials and methods}

\section{Criteria for considering studies}

Types of studies. Randomized controlled trials (RCT) with a parallel design that compared the addition of CSFs during or subsequent to myelosuppressive therapy to the no treatment or placebo regime in adult ALL patients were included. Only studies in English were accepted.

Types of participants. Adults (>19 years old) with ALL in all stages of treatment following the administration of the chemotherapy (induction, consolidation and salvage treatment) were included. Trials with participants $<15$ years old were included when separate statistics could not be obtained.

Types of interventions. The interventions were CSFs, including G-CSF or GM-CSF, administered either intravenously or subcutaneously, and concomitantly or following chemotherapy and continued for $>24 \mathrm{~h}$. CSFs were administered in a dose of $>5 \mathrm{mcg} / \mathrm{kg}$ body weight per day until absolute neutrophil counts reached $>0.5 \times 10^{9} / 1$. The studies using CSF in the priming of chemotherapy or the bone marrow transplantation were excluded.

\section{Types of outcome measures}

Primary outcomes. The primary outcome was mortality at the end of follow-up.

Secondary outcomes. Secondary outcomes were mortality at day 30 (usually parallels mortality associated with ALL induction treatment), number of patients achieving CR, number of patients with infection or severe infection and duration of neutropenia (median days).

Search methods for identification of studies. A comprehensive search was conducted between 1966 and 2014 using databases, including the Cochrane Central Register of Controlled Trials, PubMed, Web of Science and SinoMed. In PubMed, the search was conducted using combinations of Medical Subject Heading (MeSH) search terms and keywords (Fig. 1). Search details included: ('Adult' [MeSH]) AND ('Precursor Cell Lymphoblastic Leukemia-Lymphoma' [MeSH] or 'acute lymphoblastic leukemia' [All fields] or 'acute lymphoblastic leukemia' [All fields]) AND ('Colony-Stimulating Factors' [MeSH] or 'Hematopoietic Cell Growth Factors' [All fields]). Other databases were queried using identical terms for keyword searching. Only studies in English were accepted.

Data collection and analysis. Two review authors independently extracted the data from the included trials. In case of any disagreement, a third review author extracted the data. A standardized form was used to extract the relevant data on the characteristics of trials and patients, intervention protocol and outcomes.

Data synthesis. The Mantel-Haenszel method (Review Manager 5.2) (19) was used to estimate the risk ratios (RR)

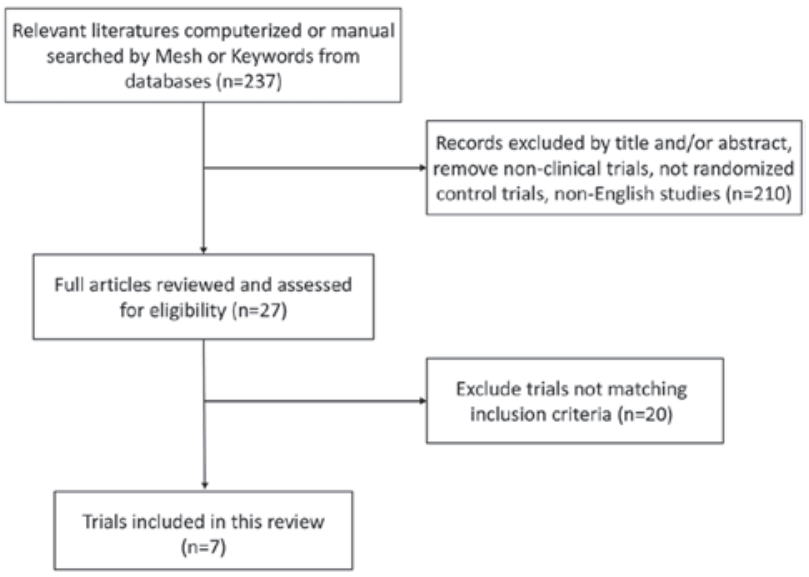

Figure 1. Flow chart of the search strategy process.

and $95 \%$ confidence intervals (CI) for dichotomous data. A fixed-effect model was used and a sensitivity analysis was performed by repeating the above analysis using a random-effects model.

\section{Results}

Description of studies. In total, 7 RCTs with 753 patients were included [Geissler et al (20), Hallbook et al (21), Holowiecki et al (22), Ifrah et al (23), Larson et al (24), Ottmann et al (25) and Thomas et al (26)]. There were only two double-blind RCTs and the remaining studies were open-label. The duration of follow-up ranged from 22 months to 8 years. The age of patients ranged from 15-79 year old. (Table I).

Risk of bias in included studies. Generation of randomization sequence, allocation concealment, blinding, incomplete outcome data and selective reporting were assessed for all 7 included trials (Fig. 2).

\section{Effects of interventions}

Primary outcomes. Five trials including 611 patients reported mortality at the end of follow-up. The end of follow-up ranged from 1 to 5 years. The addition of CSFs to chemotherapy decreased the mortality between patients treated with chemotherapy and CSFs, and those treated with chemotherapy alone. Meta-analysis showed a significant difference to the CSF group with an RR of 0.85 (95\% CI, 0.75-0.95) (Fig. 3).

Secondary outcomes. All 7 trials, including 753 patients, reported the all-cause mortality at day 30. The addition of CSFs to chemotherapy decreased the mortality at day 30 in the adult ALL patients (RR, 0.41; 95\% CI, 0.23-0.74) (Fig. 4).

Number of patients achieving CR. All 7 trials, including 753 patients, reported the CR rate. The addition of CSF to chemotherapy compared to placebo or nointervention increased the rate of CR, with an RR of 1.14 (95\% CI, 1.05-1.23) (Fig. 5).

Number of patients with infection and severe infection. A total of 4 trials reported the number of patients with infection and 2 trials reported the number of patients with 


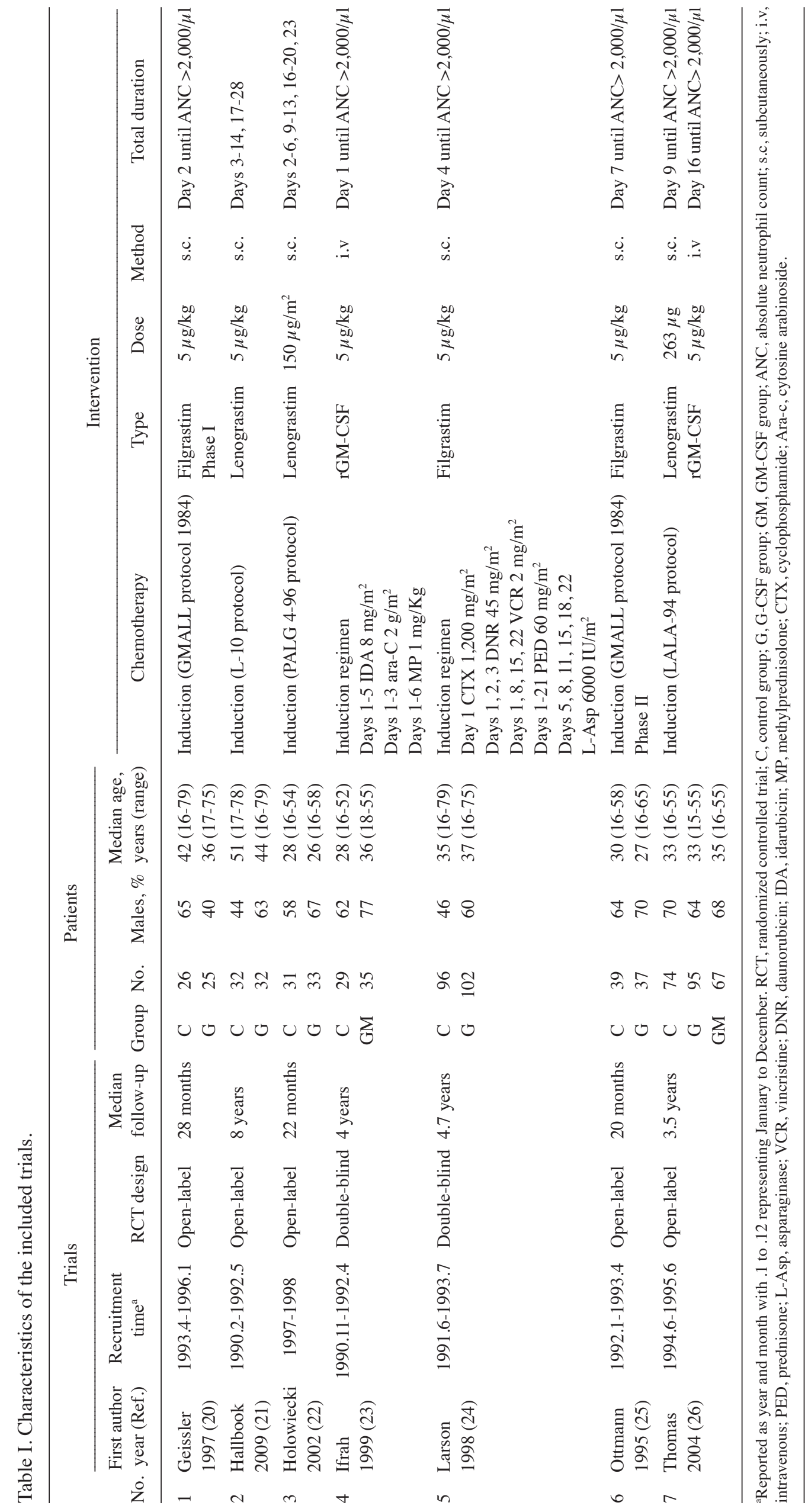


A

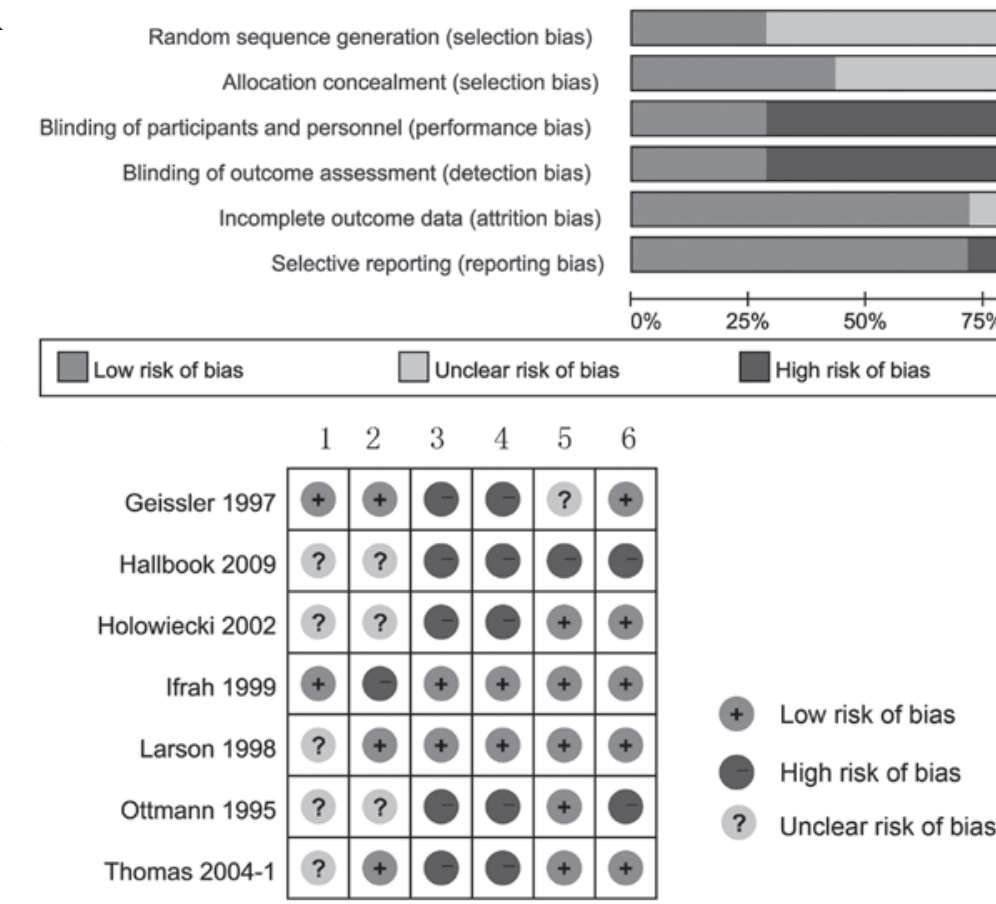

Figure 2. Risk of bias graph. Assessments regarding each risk of bias item are presented as percentages across (A) all and (B) for each of the included studies. Lanes 1, random sequence generation; 2, allocation concealment; 3, blinding of participants and personnel; 4, blinding of outcome assessment; 5 , incomplete outcome data; 6 , selective reporting.

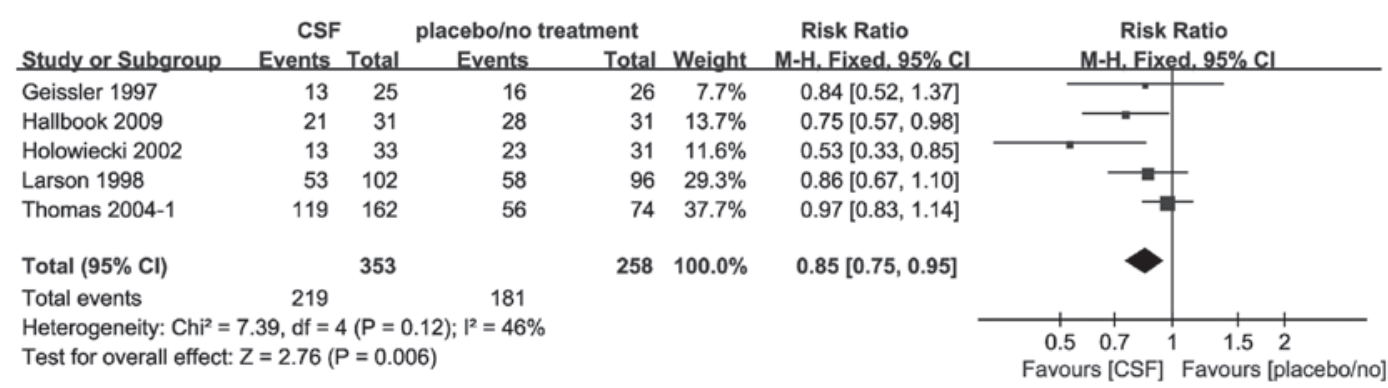

Figure 3. Forest plot of the comparison for the overall mortality at the end of follow-up. The risk ratio between the CSF and placebo groups is 0.85 (95\% CI, 0.75-0.95). $\mathrm{I}^{2}=46 \%$, showing middle heterogeneity.

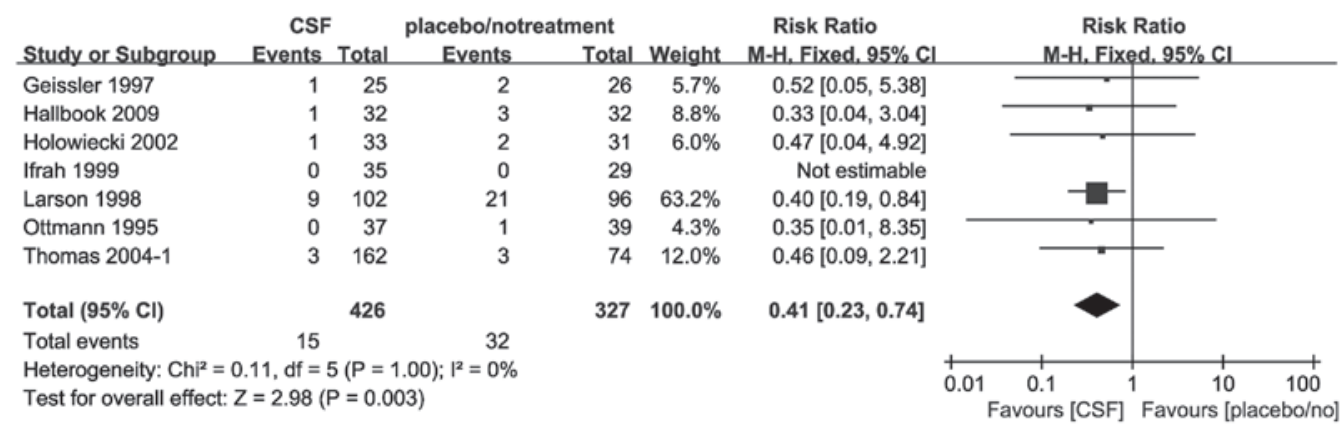

Figure 4. Forest plot of the comparison for the all-cause mortality at day 30. The risk ratio between the CSF and placebo groups is 0.41 (95\% CI, $0.23-0.74$ ). $\mathrm{I}^{2}=0$, showing no substantial heterogeneity.

severe infection (World Health Organization grade $\geq$ III). The addition of CSF to chemotherapy compared to placebo or no intervention reduced the occurrence of infection (RR, 0.8; 95\% CI, 0.7-0.9) and severe infection (RR, 0.48; 95\% CI, 0.3-0.75) (Fig. 6).
Duration of neutropenia from randomization. In total, 6 studies reported on neutropenia duration (20-22,24-26). In these studies, neutropenia was defined as $<0.5 \times 10^{9} / 1$ neutrophils. The median duration of neutropenia ranged between 8 and 17 days in the CSFs arm and between 12.5 and 24 days 


\begin{tabular}{|c|c|c|c|c|c|c|c|c|c|}
\hline Study or Subgroup & \multicolumn{2}{|c|}{ CSF } & \multicolumn{2}{|c|}{ placebo/no treatment } & Weight & $\begin{array}{c}\text { Risk Ratio } \\
M-H \text {.Fixed, } 95 \% \mathrm{Cl}\end{array}$ & \multicolumn{3}{|c|}{$\begin{array}{c}\text { Risk Ratio } \\
\text { M-H, Fixed, } 95 \% \mathrm{Cl}\end{array}$} \\
\hline Geissler 1997 & 24 & 25 & 20 & 26 & $7.5 \%$ & $1.25[1.00,1.56]$ & & & \\
\hline Hallbook 2009 & 26 & 32 & 24 & 32 & $9.2 \%$ & $1.08[0.84,1.41]$ & & & \\
\hline Holowiecki 2002 & 27 & 33 & 21 & 31 & $8.3 \%$ & $1.21[0.90,1.62]$ & & & \\
\hline Ifrah 1999 & 27 & 35 & 19 & 29 & $7.9 \%$ & $1.18[0.86,1.62]$ & & & \\
\hline Larson 1998 & 87 & 102 & 71 & 96 & $28.0 \%$ & $1.15[1.00,1.33]$ & & & \\
\hline Ottmann 1995 & 36 & 37 & 36 & 39 & $13.4 \%$ & $1.05[0.95,1.17]$ & & & \\
\hline Thomas 2004-1 & 120 & 162 & 49 & 74 & $25.7 \%$ & $1.12[0.93,1.35]$ & & & \\
\hline Total $(95 \% \mathrm{Cl})$ & & 426 & & 327 & $100.0 \%$ & $1.14[1.05,1.23]$ & & & \\
\hline Total events & 347 & & 240 & & & & & & \\
\hline $\begin{array}{l}\text { Heterogeneity: } \mathrm{Ch}^{2}{ }^{2}= \\
\text { Test for overall effect: }\end{array}$ & $\begin{array}{l}.09, \mathrm{df}=6 \\
=3.22(\mathrm{P}\end{array}$ & $\begin{array}{l}(P=0 . \\
=0.00\end{array}$ & $\begin{array}{l}0.80) ; 1^{2}=0 \% \\
01)\end{array}$ & & & & $\begin{array}{cc}1 & 1 \\
0.7 & 0.85\end{array}$ & 11.2 & 1.5 \\
\hline
\end{tabular}

Figure 5. Forest plot of the comparison for complete remission. The risk ratio between the CSF and placebo groups is 1.14 (95\% CI, $1.05-1.23)$. $\mathrm{I}^{2}=0$, showing no substantial heterogeneity.

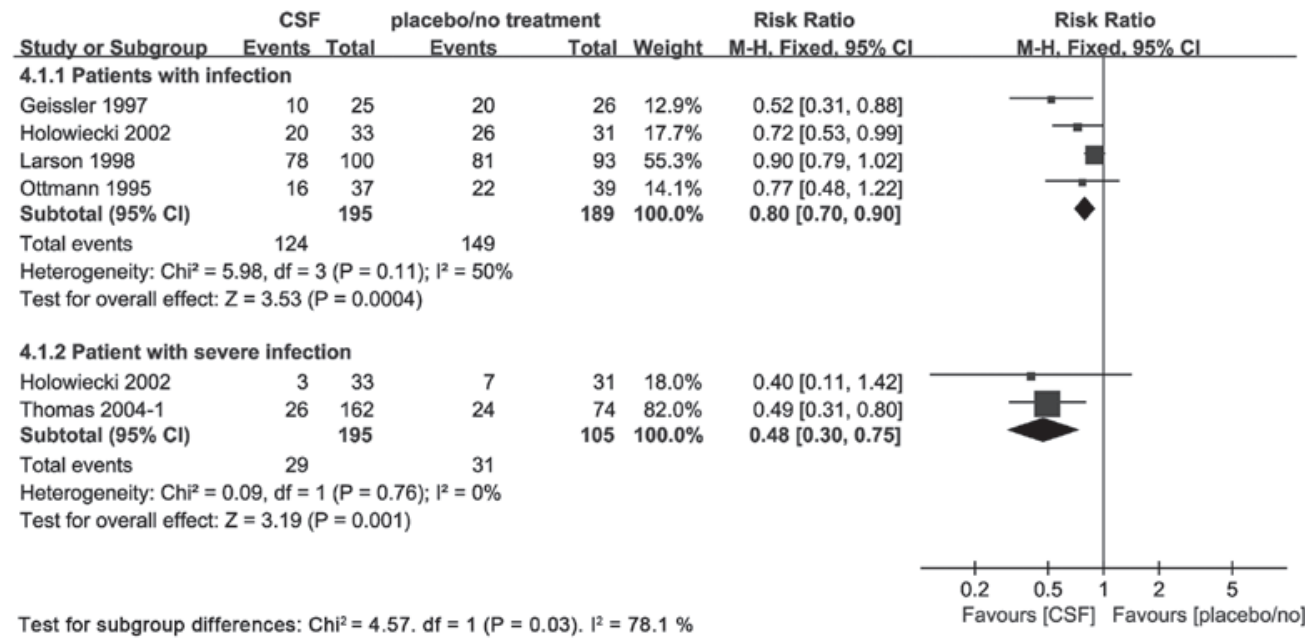

Figure 6. Forest plot of the subgroup comparison for patients with infection and severe infection. The risk ratio in the infection subgroup is 0.8 (95\% CI, $0.7-0.9)$ and in the severe infection subgroup is 0.48 (95\% CI, 0.3-0.75).

in the control arm. A meta-analysis could not be conducted on this outcome as it is a non-normally dispersed variable and outcomes were reported as medians in the majority of trials.

\section{Discussion}

CSFs are often used to prevent chemotherapy-related infection and febrile neutropenia in cancer patients. Currently, there are certain systematic reviews that investigate the effect of CSF in cancer treatment (27-29), particularly in ALL (30).

In the present study, we conclude that CSF is effective in the prevention of the myelosuppressive therapy-related infectious complications in adult ALL. CSFs were also able to improve the overall mortality. A recent meta-analysis that focused on the prophylactic use of G-CSF in ALL patients was published by Giebel et al (31). The study conducted a joint analysis and obtained a conclusion that is agreeable with the present study, which is that the prophylactic use of G-CSF during induction of ALL is effective and associated with improved long-term outcome (31).

An important issue is that based on the current available evidence and physician experience, the optimum application of CSF in clinical practice is unknown.

Five of the included studies used G-CSF and two used GM-CSF (20-26). The commonly used dosage of G-CSF and
GM-CSF is $5 \mu \mathrm{g} / \mathrm{kg}$. A subgroup meta-analysis to compare the difference was conducted, however, the number of studies included was small.

Another issue is the optimal injection scheme of CSF. The initial time varies from 2 to 9 days after the chemotherapy. The end time is until the absolute neutrophil count recovery (ANCR), however the criteria are different. Certain studies identified ANCR as ANC 1,500/ $\mu$ l, and others identified it as $>2,000 / \mu 1$. Until now, there is no consensus of CSF in the clinical use.

In all the included studies, the administration of CSF was only used in the induction phase of chemotherapy. However, in the treatment of adult ALL, the protocol is often composed of the induction, intensification, re-intensification and maintenance phase. The effect and safety of CSF following a long-term treatment is unknown, which is used in every course of chemotherapy. In current studies, the identification of receptors to G-CSF, GM-CSF and interleukin-3 (32) on the membrane of leukemic lymphoblasts was described. Certain initial molecular studies identified growth of ALL blast cell colonies following exposure to G-CSF (33), GM-CSF and other cytokines (such as interleukin) (34) in in vitro assays. It is possible that the frequent use of CSF during each myelosuppressive therapy can influence the prognosis of ALL regarding $\mathrm{CR}$, relapse and overall mortality rates. 
Additionally, the number of included trials is small and there are no large sample trials. The majority of the studies are open-label studies, which are known as subject to potential risk of performance and detection bias. In another aspect, there are only 7 published studies included in the present study, and the unpublished studies could not be identified. Therefore, the publication bias is significant. Furthermore, there are certain RCTs that were supported by pharmaceutical company funding, which has an impact on the quality of studies. Pharmaceutical company funding is empirically linked to potential bias. There is a need to avoid pharmaceutical funding as much as possible to minimize results and publication bias (35).

Future RCTs should focus on determination of the optimal CSF dosage and the timing point, as well as the use of CSFs during all cycles of the most intensive phases of treatment. As CSFs are a relatively expensive drug, cost-effectiveness analysis of the implication of routine use in ALL therapy should be performed with consideration of different economic scenarios.

In conclusion, it is recommended that CSFs can be administered to ALL patients during myelosuppressive chemotherapy, particularly in the induction phase. In statistics, the administration of CSF reduces the mortality at the end of follow-up and at day 30 , decreases the occurrence of infections and shortens the duration of neutropenia. By contrast, the administration of CSF increases the CR rate following the induction course.

\section{Acknowledgements}

The present study was supported by a grant from the National Nature Science Foundation of China (grant no. 81270615).

\section{References}

1. Jabbour EJ, Faderl S and Kantarjian HM: Adult acute lymphoblastic leukemia. Mayo Clinic Proc 80: 1517-1527, 2005.

2. Siegel R, Naishadham D and Jemal A: Cancer statistics, 2013. CA Cancer J Clin 63: 11-30, 2013.

3. Hiroto Inaba MG and Charles G Mullighan: Acute lymphoblastic leukaemia. Lancet 381: 1943-1955, 2013.

4. Esparza SD and Sakamoto KM: Topics in pediatric leukemia-acute lymphoblastic leukemia. Med Gen Med 7: 23 , 2005.

5. Pui $\mathrm{CH}$ and Evans WE: Acute lymphoblastic leukemia. N Engl J Med 339: 605-615, 1998.

6. Pui CH, Pei D, Sandlund JT, et al: Long-term results of St Jude Total Therapy Studies 11, 12,13A, 13B and 14 for childhood acute lymphoblastic leukemia. Leukemia 24: 371-382, 2010.

7. Gaynon PS, Angiolillo AL, Carroll WL, et al: Long-term results of the children's cancer group studies for childhood acute lymphoblastic leukemia 1983-2002: a Children's Oncology Group Report. Leukemia 24: 285-297, 2010.

8. Linker C, Damon L, Ries C and Navarro W: Intensified and shortened cyclical chemotherapy for adult acute lymphoblastic leukemia. J Clin Oncol 20: 2464-2471, 2002.

9. Kantarjian H, Thomas D, O'Brien S, et al: Long-term follow-up results of hyperfractionated cyclophosphamide, vincristine, doxorubicin and dexamethasone (Hyper-CVAD), a dose-intensive regimen, in adult acute lymphocytic leukemia. Cancer 101: 2788-2801, 2004.

10. Rowe JM, Buck G, Burnett AK, et al: Induction therapy for adults with acute lymphoblastic leukemia: results of more than 1500 patients from the international ALL trial: MRC UKALL XII/ECOG E2993. Blood 106: 3760-3767, 2005.

11. Bassan R and Hoelzer D: Modern therapy of acute lymphoblastic leukemia. J Clin Oncol 29: 532-543, 2011.

12. Annino L, Vegna ML, Camera A, et al: Treatment of adult acute lymphoblastic leukemia (ALL): long-term follow-up of the GIMEMA ALL 0288 randomized study. Blood 99: 863-871, 2002.
13. Larson RA, Dodge RK, Burns CP, et al: A five-drug remission induction regimen with intensive consolidation for adults with acute lymphoblastic leukemia: cancer and leukemia group B study 8811. Blood 85: 2025-2037, 1995.

14. Takeuchi J, Kyo T, Naito K, et al: Induction therapy by frequent administration of doxorubicin with four other drugs, followed by intensive consolidation and maintenance therapy for adult acute lymphoblastic leukemia: the JALSG-ALL93 study. Leukemia 16: 1259-1266, 2002.

15. Thomas X, Boiron JM, Huguet F, et al: Outcome of treatment in adults with acute lymphoblastic leukemia: analysis of the LALA-94 trial. J Clin Oncol 22: 4075-4086, 2004.

16. Griffin JD: Hematopoietic growth factors. In: Cancer: Principles and Practice of Oncology. DeVita VT Jr, Hellman S and Rosenburg SA (eds). Vol 1. 6th edition. Lippincott Williams \& Wilkins, Philadelphia, PA, pp2798-2809, 2001.

17. Petros WP: Colony-stimulating factors. In: Cancer Chemotherapy and Biotherapy: Principles and Practice. Chabner BA and Longo DL (eds). 5th edition. Lippincott Williams \& Wilkins, Philadelphia, PA, pp357-392, 2001.

18. Ozer H, Armitage JO, Bennett CL, et al: 2000 update of recommendations for the use of hematopoietic colony-stimulating factors: evidence-based, clinical practice guidelines. American Society of Clinical Oncology Growth Factors Expert Panel. J Clin Oncol 18: 3558-3585, 2000.

19. The Cochrane Collaboration: Review Manager (RevMan) 5.2 http://tech.cochrane.org/revman/download. Accessed December 25, 2013.

20. Geissler K, Koller E, Hubmann E, et al: Granulocyte colony-stimulating factor as an adjunct to induction chemotherapy for adult acute lymphoblastic leukemia-a randomized phase-III study. Blood 90: 590-596, 1997.

21. Hallbook H, Bjorkholm M, Hagglund H and Smedmyr B: Does granulocyte colony-stimulating factor improve long-term outcome in adult acute lymphoblastic leukemia? Leuk Lymphoma 50: 1872-1874, 2009.

22. Holowiecki J, Giebel S, Krzemien S, et al: G-CSF administered in time-sequenced setting during remission induction and consolidation therapy of adult acute lymphoblastic leukemia has beneficial influence on early recovery and possibly improves long-term outcome: a randomized multicenter study. Leuk Lymphoma 43: 315-325, 2002.

23. Ifrah N, Witz F, Jouet JP, et al: Intensive short term therapy with granulocyte-macrophage-colony stimulating factor support, similar to therapy for acute myeloblastic leukemia, does not improve overall results for adults with acute lymphoblastic leukemia. GOELAMS Group. Cancer 86: 1496-1505, 1999.

24. Larson RA, Dodge RK, Linker CA, et al: A randomized controlled trial of filgrastim during remission induction and consolidation chemotherapy for adults with acute lymphoblastic leukemia: CALGB study 9111. Blood 92: 1556-1564, 1998.

25. Ottmann OG, Hoelzer D, Gracien E, et al: Concomitant granulocyte colony-stimulating factor and induction chemoradiotherapy in adult acute lymphoblastic leukemia: a randomized phase III trial. Blood 86: 444-450, 1995.

26. Thomas X, Boiron JM, Huguet F, et al: Efficacy of granulocyte and granulocyte-macrophage colony-stimulating factors in the induction treatment of adult acute lymphoblastic leukemia: a multicenter randomized study. Hematol J 5: 384-394, 2004.

27. Bohlius J, Herbst C, Reiser M, Schwarzer G and Engert A: Granulopoiesis-stimulating factors to prevent adverse effects in the treatment of malignant lymphoma. Cochrane Database Syst Rev: CD003189, 2008.

28. Clark OA, Lyman G, Castro AA, Clark LG and Djulbegovic B: Colony stimulating factors for chemotherapy induced febrile neutropenia. Cochrane Database Syst Rev: CD003039, 2003.

29. Renner P, Milazzo S, Liu JP, Zwahlen M, Birkmann J and Horneber M: Primary prophylactic colony-stimulating factors for the prevention of chemotherapy-induced febrile neutropenia in breast cancer patients. Cochrane Database Syst Rev: CD007913, 2012.

30. Gurion R, Belnik-Plitman Y, Gafter-Gvili A, et al: Colony-stimulating factors for prevention and treatment of infectious complications in patients with acute myelogenous leukemia. Cochrane Database Syst Rev: CD008238, 2011.

31. Giebel S, Thomas X, Hallbook H, et al: The prophylactic use of granulocyte-colony stimulating factor during remission induction is associated with increased leukaemia-free survival of adults with acute lymphoblastic leukaemia: a joint analysis of five randomised trials on behalf of the EWALL. Eur J Cancer 48: 360-367, 2012. 
32. Inukai T, Sugita K, Iijima K, et al: Leukemic cells with 11q23 translocations express granulocyte colony-stimulating factor (G-CSF) receptor and their proliferation is stimulated with G-CSF. Leukemia 12: 382-389, 1998.

33. Benko I, Kovacs P, Szegedi I, et al: Effect of myelopoietic and pleiotropic cytokines on colony formation by blast cells of children with acute lymphoblastic leukemia. Naunyn-Schmiedeberg's Arch Pharmacol 363: 499-508, 2001.
34. Gattei V, Aldinucci D, Attadia V, et al: Human granulocyte-macrophage colony-stimulating factor supports the clonogenic growth of B-lineage acute lymphoblastic leukemias expressing myeloid antigens. Cytokines Cell Mol Ther 3: 141-151, 1997.

35. Lexchin J, Bero LA, Djulbegovic B and Clark O: Pharmaceutical industry sponsorship and research outcome and quality: systematic review. BMJ 326: 1167-1170, 2003. 